\title{
POLYPHASE ADAPTIVE FILTER BANKS FOR SUBBAND DECOMPOSITION
}

\author{
Ömer N. Gerek, A. Enis Çetin \\ Bilkent University, Dept. of Electrical and Electronics Engineering, \\ Bilkent, Ankara TR-06533, Turkey \\ E-mail: gerek@ee.bilkent.edu.tr \\ Phone: (90) 312-266 4307 Fax: (90) 312-266 4126
}

\begin{abstract}
Subband decomposition is widely used in signal processing applications including image and speech compression. In most practical cases, the goal is to obtain subband signals that are suitable for data compression. In this paper, we present Perfect Reconstruction (PR) polyphase filter bank structures in which the filters adapt to the changing input conditions. This leads to higher compression results for images containing sharp edges, text, and subtitles.
\end{abstract}

\section{ADAPTIVE PREDICTION FILTERS IN POLYPHASE FORM}

Consider the simple two band PR polyphase decomposition structure shown in Figure 1 in which the filter $P_{1}$ can be

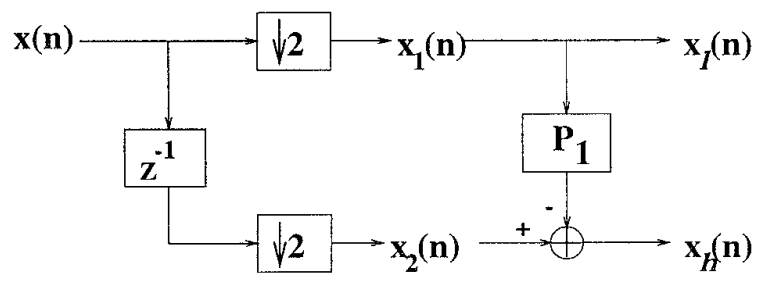

Figure 1: Polyphase analysis and synthesis structure

either linear or nonlinear [1],[2]. This structure has a simple transform matrix:

$$
\mathbf{P}=\left[\begin{array}{cc}
1 & -P_{1}(.) \\
0 & 1
\end{array}\right]
$$

A good $P_{1}$ filter would be the one that can predict the samples of $x_{2}(n)$ as close as possible. In binary image coding, successful coding results are obtained by selecting $P_{1}$ as a median filter [3]. In [4], the $P_{1}$ is chosen as a. FIR low-pass and a median filter pair which are interchanged according to characteristics of the input signal. In this paper, the adaptive FIR filters and adaptive order statistics filters are used in the filter bank and image coding algorithms are developed based on the resulting structure. In all cases, the inverse of the $\mathbf{P}$ matrix in Eq. (1) is given as:

$$
\mathbf{P}^{-1}=\left[\begin{array}{cc}
1 & P_{1}(.) \\
0 & 1
\end{array}\right]
$$

A predictor should be adaptive for image and video signals as they are unstationary in nature. This reasoning

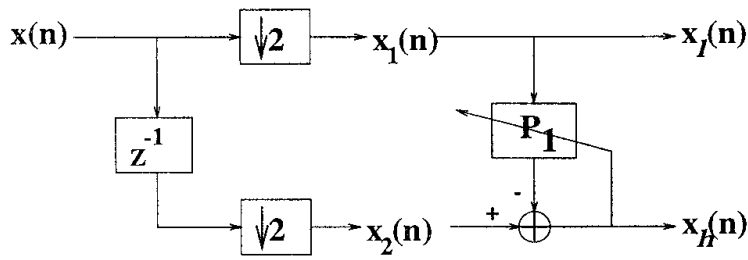

Figure 2: Adaptive structure analysis stage

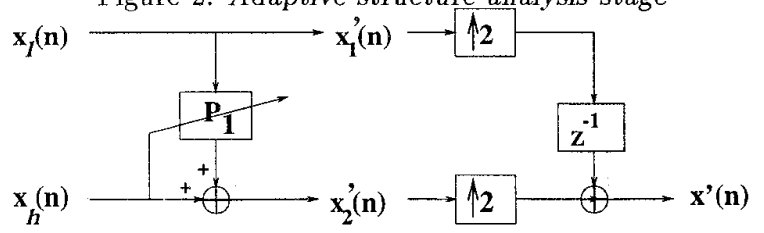

Figure 3: Adaptive structure synthesis stage

leads to the polyphase structure shown in Fig. 2 in which the prediction filter adapts itself to minimize the highband signal $x_{h}(n)$. In this way, unnecessary information in $x_{2}(n)$ is removed and an uncorrelated signal is transmitted to the receiver. This is especially useful when there are sharp transition regions in an image such as subtitles, text and graphics.

The polyphase adaptive filter bank structure is shown in Figures 2, 3. In this figure, the signal $x_{2}(n)$ is predicted from $x_{1}(n)$ (or from the even samples of $x(n)$ ) as follows:

$$
\hat{x}_{2}(n)=\sum_{k=-N}^{N} a_{k} x_{1}(n-k)=\sum_{k=-N}^{M} a_{k} x(2 n-2 k)
$$

where the filter coefficients $a_{k}$ 's are updated using an LMStype algorithm [5],[6], and the subsignal $x_{h}$ is given by

$$
x_{h}(n)=x_{2}(n)-\mathbf{P}_{1}\left(x_{1}\right)=x_{2}(n)-\hat{x}_{2}(n) .
$$

Since $x_{h}(n)$ corresponds to the error signal in the adaptation algorithm, the decoder can also update the filter coeffcients without any side information. It can easily be shown that the PR property is preserved as long as the same adaptation strategy is used in both encoding and decoding.

Another choice for $P_{1}$ is the adaptive Order Statistics (OS) filter [7]-[10]. Similar to the adaptive FIR case, the OS filters can be adapted by minimizing the subsignal $x_{h}$ using an adaptation strategy. 


\section{MULTICHANNEL EXTENSIONS OF THE BASIC ADAPTIVE FILTER BANK}

The structure in Fig. 1 and 2 can be extended to contain cascaded PR blocks as follows:

$\mathbf{P}=\left[\begin{array}{cc}1 & -P_{1}(.) \\ 0 & 1\end{array}\right] \times\left[\begin{array}{cc}1 & 0 \\ G_{1}(.) & 1\end{array}\right] \times\left[\begin{array}{cc}1 & -P_{2}(.) \\ 0 & 1\end{array}\right] \times \cdots$

where the filters $P_{1}, G_{1}, P_{2}, \cdots$ can be linear, nonlinear or adaptive. In this way, the upper and lower branch subsignals can be filtered a number of times. The inverse matrix is given as

$\mathbf{P}^{-1}=\cdots \times\left[\begin{array}{cc}1 & P_{2}(.) \\ 0 & 1\end{array}\right] \times\left[\begin{array}{cc}1 & 0 \\ -G_{1}(.) & 1\end{array}\right] \times\left[\begin{array}{cc}1 & P_{1}(.) \\ 0 & 1\end{array}\right]$

Multichannel and multidimensional extensions are also possible in a straightforward manner. An example of the $\mathrm{PR}$ multichannel extension is illustrated in Fig. 4. In this

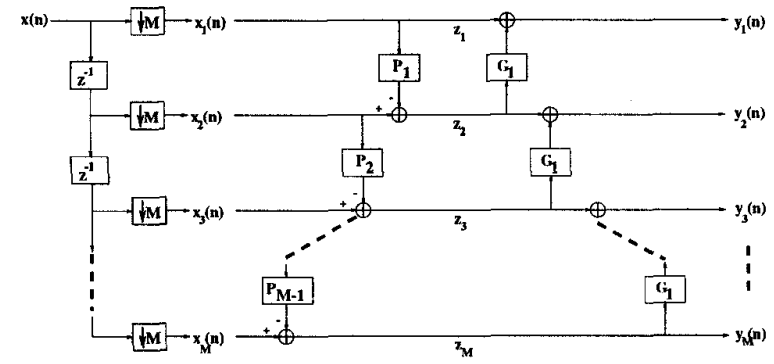

Figure 4: Multi-band analysis structure

figure, an $\mathrm{M}$ band decomposition with two cascaded $\mathrm{PR}$ building blocks is illustrated. The PR property of this structure can be proved easily. In the analysis stage,

$$
\begin{aligned}
& z_{1}=x_{1} \\
& z_{i}=x_{i}-P_{i-1}\left(z_{i-1}\right), \quad i=2,3, \ldots, M \\
& y_{i}=z_{i}+G_{i}\left(z_{i+1}\right), \quad i=1,2, \ldots, M-1 \\
& y_{M}=z_{M}
\end{aligned}
$$

The corresponding $\mathbf{P}$ matrix for this example can be given as :

$$
\left[\begin{array}{ccccc}
1 & -P_{1} & 0 & 0 & \cdots \\
0 & 1 & -P_{2} & 0 & \cdots \\
0 & 0 & 1 & -P_{3} & \cdots \\
\vdots & \vdots & \vdots & \ddots & \ddots
\end{array}\right] \times\left[\begin{array}{ccccc}
1 & 0 & 0 & 0 & \cdots \\
G_{1} & 1 & 0 & 0 & \cdots \\
0 & G_{2} & 1 & 0 & \cdots \\
\vdots & \vdots & \ddots & \ddots & \ddots
\end{array}\right]
$$

The PR can be achieved with any choice of the nonlinear operators as the $\mathbf{P}$ matrix can be inverted regardless of the filters $P_{i}$ 's and $G_{i}$ 's. This leads to the following synthesis equations:

$$
\begin{aligned}
& z_{M}^{\prime}=z_{M} \\
& z_{i}^{\prime}=y_{i}-G_{i}\left(z_{i+1}^{\prime}\right)=z_{i}, \quad i=M-1, \ldots, 1 \\
& x_{1}^{\prime}=z_{1}=x_{1} \\
& x_{i}^{\prime}=z_{i}^{\prime}+P_{i-1}\left(z_{i-1}^{\prime}\right)=z_{i}+P_{i-1}\left(z_{i-1}\right)=x_{i}
\end{aligned}
$$

The outputs, $x_{i}^{\prime}$, of the synthesis filters are the same as the polyphase components, $x_{i}$, of the analysis filter bank. Notice that there are no restrictions on the filters $P_{i}$ and $G_{i}$ for perfect reconstruction.

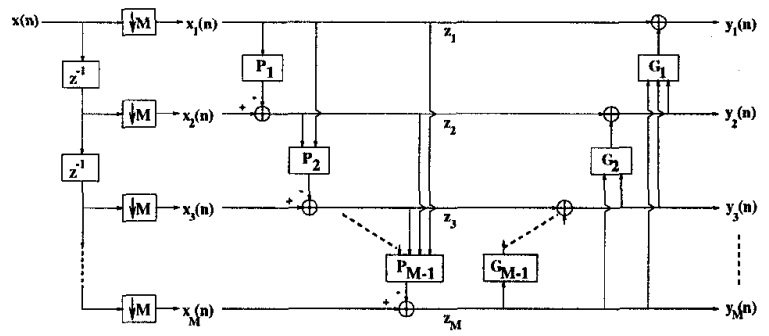

Figure 5: Multi-band analysis structure - 2

Another multichannel extension structure is illustrated in Fig. 5. The previous structure was considering the nearest polyphase component value of the input data stream. However, the structure in Fig. 5 is using increasing amount of polyphase component values for prediction as you go from up to down in the figure. The analysis and synthesis equations for this structure can be given as follows.

Analysis:

$$
\begin{aligned}
& z_{1}=x_{1} \\
& z_{i}=x_{i}-P_{i-1, i-1}\left(-P_{i-2, i-2}\left(-P_{i-3, i-3}\left(\cdots\left(-P_{1,1}\right)\right)\right)\right) \\
& \text { - } P_{i-1, i-2}\left(-P_{i-3, i-3}\left(\cdots\left(-P_{1,1}\right)\right)\right) \\
& \vdots \vdots \\
& \text { - } P_{i-1,1}, \quad i=2,3, \ldots, M \\
& =x_{i}-P_{i-1}\left(z_{1}, z_{2}, \ldots, z_{i-1}\right) \\
& \begin{array}{l}
y_{M}=z_{M} \\
y_{i}=z_{i}+G_{i}\left(y_{M}, \ldots, y_{i+1}\right), \quad i=1,2, \ldots, M-1
\end{array}
\end{aligned}
$$

Synthesis:

$$
\begin{aligned}
z_{i}^{\prime}=y_{i}-G_{i}\left(y_{M}, \ldots, y_{i+1}\right) & =z_{i}, \quad i=1,2, \ldots, M-1 \\
z_{M}^{\prime}=y_{M} & =z_{M} \\
x_{1}^{\prime}=z_{1} & =x_{1} \\
x_{i}^{\prime}=z_{i}+P_{i-1}\left(z_{1}, z_{2}, \ldots, z_{i-1}\right. & =x_{i}, \quad i=2,3, \ldots, M
\end{aligned}
$$

This later structure also yields analysis matrices which can be composed to upper and lower triangular matrices with elements containing $P_{i}$ 's and $G_{i}$ 's only. In this structure, for predicting $z_{i}$ 's, the number of data used is increased with increasing index $i$. Conversely, more $z_{i}$ samples are used for predicting $y_{i}$ 's when the index $i$ is small. The computational and analysis complexity of this structure is high as compared to the structure in Fig. 4. Furthermore, no coding gain over the structure in Fig. 5 has been observed for the structure in Fig. 4.

\section{CODING GAIN ANALYSIS}

The structure in Fig. 2 can be considered as a transformation on the input signals $x_{1}$ and $x_{2}$. For an appropriate transform, the goal is to minimize the total variance of the transform coefficients. It has been shown in [12] that if the 
coefficients before transformation are quantized at an average bit rate $R$, the PCM quantization variance can be stated as

$$
\sigma_{r, P C M}^{2}=\epsilon^{2} 2^{-2 R} \sigma_{x}^{2}
$$

where $\epsilon^{2}$ is a scaling factor proportional to the quantizer performance factor [12], and $\sigma_{x}^{2}$ is the variance of the input signal. Similarly, if the transform coefficients are also quantized at the same rate, $R$, then the minimum transform domain quantization variance becomes

$$
\sigma_{r, T C}^{2}=\epsilon^{2} 2^{-2 R}\left(\sigma_{1} \sigma_{2}\right)
$$

The ratio of $\sigma_{r, P C M}^{2}$ to $\sigma_{r, T C}^{2}$ gives the coding gain:

$$
G_{T C}=\frac{\sigma_{x}^{2}}{\left(\sigma_{1}^{2} \sigma_{2}^{2}\right)^{(1 / 2)}}
$$

The maximization of the gain term is equivalent to min imization of the variances of the transform domain coefficients. The transform matrix in Eq. (1) shows that transform domain output $x_{1}$ corresponding to the input $x_{1}$ is identically equal to the input signal. In this case, we can maximize the coding gain only by minimizing the variance of $\mathrm{x}_{\mathrm{h}}$.

The adaptive LMS algorithm minimizes the mean squared error at the output, hence it minimizes the variance $\sigma_{2}^{2}$. This minimization, therefore, maximizes the coding gain at each sample point. Since our OS adaptive algorithm also uses LMS type adaptation, the same variance minimization arguments hold.

In our simulations, the normalized LMS algorithm is used with normalization factor $\left\|\mathrm{x}_{1}\right\|^{2}$. With different choices of the norms $\left(L^{1}, L^{2}\right.$, and $\left.L^{\infty}\right)$, different convergence performances are observed [13]. The $L^{1}$ and $L^{2}$ norms proved to perform good for most images.

\section{SIMULATION STUDIES}

The two dimensional extension of the proposed scheme is obtained by separately applying the one dimensional adaptive filter bank to the image data in a separable manner. The filter support, however, is chosen to exploit two dimensional characteristics better. The filter support is illustrated in Fig. 6.

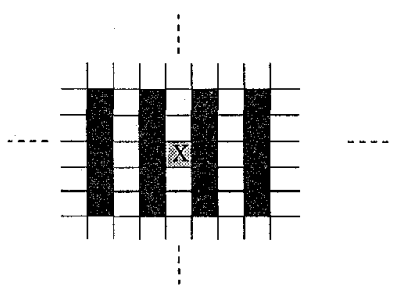

Figure 6: Two dimensional separable prediction.

In all of the simulations, we used the Embedded ZeroTree (EZT) coder to encode the transform coefficients [14]. Due to the characteristics of this coder, the best coding results were obtained by tree-structured two-band decompositions.
At compression ratios from $1: 1$ to $30: 1$, the adaptive algorithm produces acceptable outputs at the synthesis stage. Especially for the images with sharp variations, this algorithm gives better PSNRs at CR from 1: to 15:1. Due to corrupting the synthesis filter adaptation at lower bit rates, the reconstructed image starts getting deteriorated more than the conventional wavelet quantization after compression ratios of 15:1. The synthesis stage starts to diverge after the CRs around $30: 1$. The CR/PSNR plot for the adaptive algorithm applied to the first image in Table 1 is shown in Fig 7. A quick remedy to avoid the divergence of

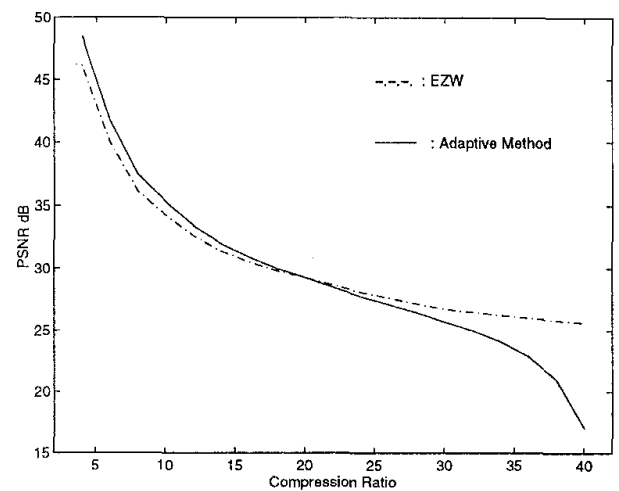

Figure 7: EZW versus adaptive method at different $C R$ 's

the the synthesis stage at very low bit rates is to substitute a fixed filter in the place of the adaptive filter whenever the bit rate is too low.

The $672 \times 560$ "barbara" image was compressed to 1 bits/pixel at a PSNR of $35.88 \mathrm{~dB}$ with the adaptive OS type prediction filter. This PSNR is also comparable to the conventional EZW compression scheme which produces $35.90 \mathrm{~dB}$ PSNR. Furthermore, our algorithm produces perceprtually better images with eliminated ringing effects at the edges.

A set of various images have been compressed using the adaptive algorithm. Some of these images are presented in Table 1.

The coding results of these images in Table 1 at $1 \mathrm{bpp}$ are presented in table 2 .

On the average, our algorithm outperforms the EZW for images with sharp variations. More robust adaptation algorithms for lower bit rates will be studied as a future work. 


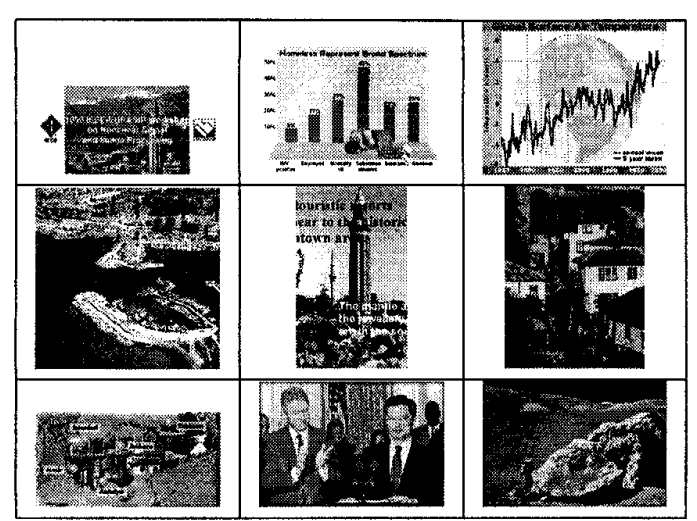

Table 1: Test images.

\begin{tabular}{|c|c|c|c|}
\hline Image Description & EZW & Adaptive FIR & Adaptive OS \\
\hline Call_for_papers(*) & 36.96 & 36.87 & 36.99 \\
\hline Sci_Tech1( $\left.{ }^{*}\right)$ & 36.41 & 36.33 & 36.48 \\
\hline Sci_Tech2(*) & 31.61 & 31.60 & 31.65 \\
\hline Tourism1 & 30.18 & 30.12 & 30.19 \\
\hline Tourism2 $\left(^{*}\right)$ & 28.02 & 27.92 & 28.07 \\
\hline Tourism3 & 32.23 & 32.18 & 32.31 \\
\hline TR_map( $\left.{ }^{*}\right)$ & 31.86 & 31.70 & 31.92 \\
\hline News0 & 34.14 & 34.15 & 34.16 \\
\hline News1 & 32.19 & 32.05 & 32.23 \\
\hline
\end{tabular}

Table 2: Experiment results (PSNR) of test images at 1 bpp.

\section{REFERENCES}

[1] F. J. Hampson and J. C. Pesquet, "A nonlinear subband decomposition with perfect reconstruction," IEEE Int. Symp. on Image Proc. 1996.

[2] Ömer N. Gerek, Metin Nafi Gürcan, A. Enis Cetin, "Binary Morphological Subband Decomposition For Image Coding," IEEE Int. Symp. on Time-Frequency and Time Scale Analysis, 1996.

[3] Ömer N. Gerek, A. Enis Çetin, "Binary nonlinear subband decomposition structures for textual image compression," Int. Symp. on Nonlinear Signal and Image Processing, NSIP'97, Sep. 1997.

[4] O. Egger, W. Li, and M. Kunt, "High Compression Image Coding Using an Adaptive Morphological Subband Decomposition," Proc IEEE, vol. 83, no. 2, pp.272-287, Feb. 1995.

[5] O. Arıkan, A. E. Çetin, Engin Erzin, 'Adaptive Filtering for non-Gaussian stable processes,' IEEE Signal Processing Letters, vol. 1, No. 11, pp. 163-165, November 1994.

[6] Gül Aydın, O. Tanrıkulu, A. Enis Çetin, "Robust least mean mixed norm adaptive filtering algorithms for $\alpha$-stable random processes," IEEE- ISCAS'97, Hong Kong, June 1997.

[7] Gonzalo. R. Arce and M. Tian, "Order-statistic filter banks," IEEE Transactions on Image Processing, 5, June 1996.

[8] I. Pitas and A. Venetsanopoulos, "Adaptive filters based on order statistics," IEEE Trans. Signal Processing, vol. 39, Feb. 1991.

[9] P. Salembier, "Adaptive rank order based filters," EURA SIP Signal Processing, 27(1):1-25, April 1992.

[10] P. Salembier and L. Jaquenoud, "Adaptive morphological multiresolution decomposition," In Dougherty Gader, editor, Image Algebra and Mathematical Morphology, volume 1568, pages 26-37, San Diego, USA, July 1991.

[11] S-M. Phoong, C. W. Kim, P.P Vaidyanathan, R. Ansari, "A new class of two channel biorthogonal filter banks and wavelet bases," IEEE Trans. Signal Proc., Vol.43, No.3, pp. 649-665, March 1995.

[12] N. S. Jayant, P. Noll, Digital Coding of Waveforms, Englewood Cliffs, NJ, Prentice Hall, 1984.

[13] S. C. Douglas, "A family of normalized LMS algorithms," Signal Processing LEtters, vol. 1, no.3, pp. 49-51, Mar. 1994.

[14] J. M. Shapiro, "Embedded Image Coding Using Zerotrees of Wavelet Coefficients," IEEE Trans. on Signal Processing, vol. 41, no. 12, pp. 3445 - 3462, Dec. 1993. 